\title{
Measurement of the Kaonic Hydrogen X-Ray Spectrum
}

\author{
G. Beer, ${ }^{1}$ A. M. Bragadireanu, ${ }^{2,3}$ M. Cargnelli, ${ }^{4}$ C. Curceanu-Petrascu, ${ }^{2}$ J.-P. Egger, ${ }^{5}$ H. Fuhrmann, ${ }^{4}$ C. Guaraldo, ${ }^{2}$ \\ M. Iliescu, ${ }^{2,3}$ T. Ishiwatari, ${ }^{4}$ K. Itahashi, ${ }^{6}$ M. Iwasaki, ${ }^{6}$ P. Kienle,,${ }^{4,7}$ T. Koike, ${ }^{6}$ B. Lauss, ${ }^{8}$ V. Lucherini, ${ }^{2}$ L. Ludhova, ${ }^{9}$ \\ J. Marton, ${ }^{4}$ F. Mulhauser, ${ }^{9}$ T. Ponta, ${ }^{3}$ L. A. Schaller, ${ }^{9}$ R. Seki, ${ }^{10,11}$ D. L. Sirghi, ${ }^{2,3}$ F. Sirghi, ${ }^{2}$ and J. Zmeskal ${ }^{4}$
}

(DEAR Collaboration)

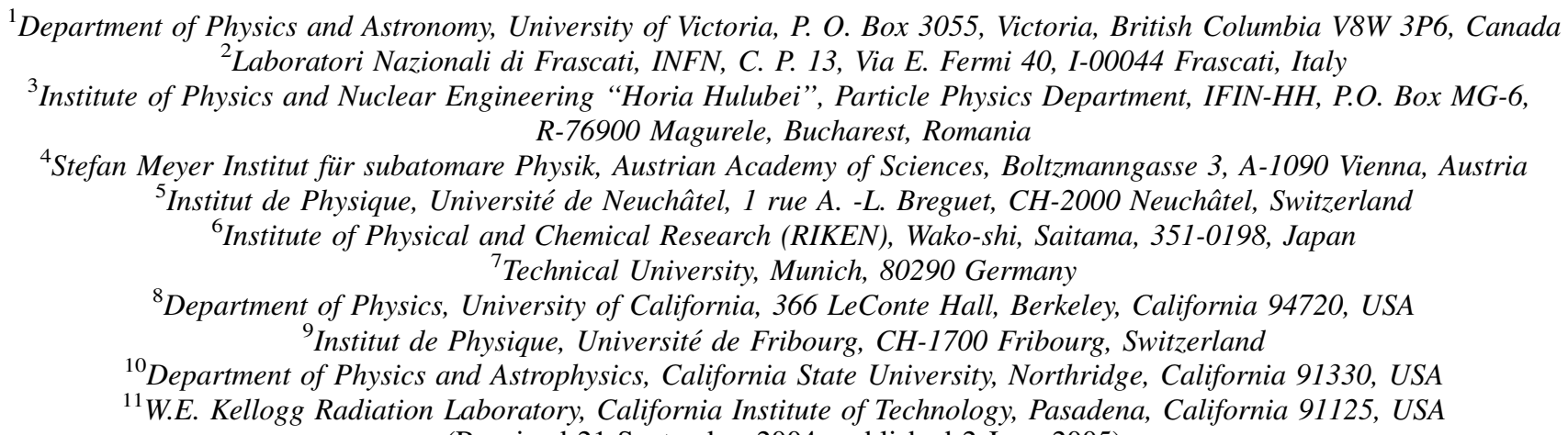

(Received 21 September 2004; published 2 June 2005)

\begin{abstract}
The DEAR (DAФNE exotic atom research) experiment measured the energy of $x$ rays emitted in the transitions to the ground state of kaonic hydrogen. The measured values for the shift $\epsilon$ and the width $\Gamma$ of the $1 s$ state due to the $K^{-} p$ strong interaction are $\epsilon_{1 s}=-193 \pm 37$ (stat) \pm 6 (syst) $\mathrm{eV}$ and $\Gamma_{1 s}=$ $249 \pm 111$ (stat) \pm 30 (syst) $\mathrm{eV}$, the most precise values yet obtained. The pattern of the kaonic hydrogen $K$-series lines, $K_{\alpha}, K_{\beta}$, and $K_{\gamma}$, was disentangled for the first time.
\end{abstract}

DOI: 10.1103/PhysRevLett.94.212302

PACS numbers: 13.75.Jz, 25.80.Nv, 36.10.Gv

Over 40 years, chiral symmetry breaking has been recognized as the essential aspect of nuclear low-energy phenomena. The outline of how the breaking plays a vital role is well known, yet its detailed dynamics is uncertain. The existence of the eight pseudoscalar mesons $(\pi, K, \eta)$ is believed to arise from spontaneous symmetry breaking of the flavor global symmetry represented by the group $\mathrm{SU}(3)_{L} \otimes \mathrm{SU}(3)_{R}$, which generates the mesons as Nambu-Goldstone bosons, leaving the vacuum only SU(3) symmetric [1]. Furthermore, the mass spectrum of these mesons reflects the explicit breaking of this symmetry [2]. In the quark model, the squares of the meson masses are proportional to the small current quark masses with the multiplicative factors of the chiral quark condensate in vacuum. The large mass difference between the mesons and the current quarks then suggests that the condensate is playing a significant role in the structure of the mesons [3].

A similar situation is expected to occur in the structure of baryons and to be manifested in the baryon-pseudoscalar meson interaction [4]. In this case, the corresponding relation is that the baryon sigma terms are proportional to the current quark masses with the factors of the chiral quark condensate for the baryons [3]. The sigma terms thus serve as the measure of the significance of the condensate in the structure of the baryons. Especially of interest here is how the $\mathrm{SU}(3)$ flavor symmetry is realized in this aspect of the nucleon structure, but more specifically, how high is the strangeness content of the nucleon. The resolution of these issues depends quite sensitively on the value of the kaonnucleon $(K N)$ sigma terms [5]. As the basic symmetry of $\mathrm{QCD}$ is $\mathrm{SU}(3)$, the $K N$ sigma terms play the central role in various nuclear phenomena, such as strangeness production in heavy-ion collision and chiral restoration in nucleon matter, a topic of astrophysical interest [6].

The $K N$ sigma terms are closely related to the lowenergy $K N$ and antikaon-nucleon $(\bar{K} N)$ scattering amplitudes [7], but the value of the $K N$ sigma terms continues to remain with a large uncertainty $[6,7]$ in spite of the recent efforts in lattice [8] and chiral perturbation [9] calculations, where the information on the $K N$ and $\bar{K} N$ scattering lengths is vital. In this work, we report an accurate measurement of the ground-state $\mathrm{x}$-ray transitions in kaonic hydrogen atoms. The shift and width of the atomic ground state is known to provide the most accurate information of the $K^{-}$-proton scattering length [10].

The recently performed $K p X$ experiment at KEK succeeded in observing unambigously, for the first time, a distinct $K_{\alpha}$ x-ray peak in kaonic hydrogen [11]. The $1 s$ level turned out to be shifted upwards (less bound, due to repulsive strong interaction) with respect to the pure electromagnetic value. This definitely swept out a long- 
standing "pseudopuzzle," concerning the sign of the interaction, between contradicting scattering data and some unreliable $\mathrm{x}$-ray results.

The overall statistics of the KEK experiment was insufficient to obtain a precise determination of the $s$-wave $K^{-} p$ scattering length. In view of the fundamental importance of this information the primary goal of the DEAR (DAФNE exotic atom research) experiment [12] at the $e^{+} e^{-}$collider DA $\Phi$ NE of the Frascati National Laboratories was a precise measurement of energies and widths of the $K$-series x rays of kaonic hydrogen.

The DEAR experiment took advantage of the lowenergy $(16 \mathrm{MeV})$, nearly monoenergetic $(\Delta p / p=0.1 \%)$ kaons from the decay of $\phi$ mesons resonantly produced by $e^{+} e^{-}$collisions in one of the two interaction points of DAФNE. Kaons went through the DEAR beam pipe made out of thin-walled aluminum with carbon fiber reinforcement, were degraded in energy to a few $\mathrm{MeV}$, and stopped in a gaseous hydrogen target placed about $10 \mathrm{~cm}$ above the beam pipe. A description of the setup is given in [13]. Here we give a summary.

The cylindrical cryogenic target cell had a diameter of $12.5 \mathrm{~cm}$ and a height of $14 \mathrm{~cm}$. Special care was taken to avoid materials with fluorescence $x$ rays in the energy region of interest. Therefore, only low $Z$ target materials were selected for target construction: aluminum (top plate and entrance ring), kapton (side wall and entrance window), and fiberglass (support structure). The target was operated with hydrogen at $25 \mathrm{~K}$ and 2 bar, resulting in a density of $2.1 \mathrm{~g} / 1$ (3\% of liquid hydrogen density).

The interaction region was shielded from primary collider background with lead walls, the target with extra lead-copper-aluminum layers. Thin titanium and zirconium foils were placed on the top of the target, in order to use the fluorescence lines as in-beam energy calibration.

Sixteen CCD detector chips (Marconi Applied Technologies, CCD55-30) with a total area of $116 \mathrm{~cm}^{2}$ faced the cryogenic target cell. Each chip had $1242 \times$ 1152 pixels with a pixel size of $22.5 \times 22.5 \mu \mathrm{m}$ and a depletion depth of about $30 \mu \mathrm{m}$. The working temperature of the CCD chips was stabilized at $\sim 165 \mathrm{~K}$ to achieve an energy resolution of $150 \mathrm{eV}$ at $6 \mathrm{keV}$ with a readout of an entire chip every $90 \mathrm{sec}$.

The flux of the charged kaons was measured by two plastic scintillators which detected the back-to-back emitted kaons [14]. A series of measurements with the target filled with nitrogen instead of hydrogen were performed before the kaonic hydrogen one. The aim was to optimize the kaon stopping points distribution, to measure the machine background, and to test the DEAR technique to create and detect exotic atoms by measuring $\mathrm{x}$ rays of three kaonic nitrogen transitions [13]. In the kaonic hydrogen measurement data for $58.4 \mathrm{pb}^{-1}\left(84.1 \times 10^{6} \mathrm{~K}^{-}\right)$were collected. Furthermore, a measurement with not colliding, i.e., separated $e^{+} e^{-}$beams in the interaction region, and intentionally high x-ray background was also performed. These data represent the so-called no-collision background. The clean extraction of the kaonic hydrogen $\mathrm{x}-$ ray spectrum required a careful treatment of the raw data. The applied procedure included several selection steps.

The "cluster analysis" selected single and double pixel events. This powerful background rejection is based on the fact that $\mathrm{x}$ rays in the $1-10 \mathrm{keV}$ energy region have a high probability to deposit all energy in a single or two adjacent pixels, whereas the energy deposited from charged particles, gammas, and neutrons is distributed over several pixels, (so-called "cluster events"), which can be rejected.

Even without irradiation, CCDs have a charge pedestal due to the electronic noise. This "noise peak" was analyzed in position and width at each measurement and data with broadened width were rejected. From position and width of the noise peak, a "noise threshold" was fixed: a pixel with charge above that threshold was regarded as a hit pixel. A small charge transfer inefficiency during readout creates a position dependent energy calibration. This wellknown effect was corrected in the analysis routine.

An energy calibration procedure, based on fluorescence lines from setup materials excitation, and from the Ti and $\mathrm{Zr}$ foils, was applied for each detector. Data of all individual detectors were then added. The overall resolution of the sum of detectors was determined: the values range from $130 \mathrm{eV}$ (FWHM) for Ca $K_{\alpha}(3.6 \mathrm{keV})$ to $280 \mathrm{eV}$ for $\mathrm{Zr} K_{\alpha}$ $(15.7 \mathrm{keV})$. The energy spectra consist of a continuous background component, fluorescence lines from setup materials, and kaonic hydrogen lines.

The analysis of the kaonic hydrogen $K$ lines meets 3 crucial challenges: (i) determination of the continuous background shape, to which the width of the kaonic hydrogen lines is very sensitive; (ii) disentanglement of the residual iron $K_{\alpha}$ fluorescence line (coming from the setup components) which partially overlaps the kaonic $K_{\alpha}$ signal; (iii) dealing with the high kaonic hydrogen $K$-series transitions 5-1 and higher $\left(K_{\text {high }}\right)$, which cannot be resolved and have poorly known yields.

In order to deal at best with these challenges, two independent analyses were performed. Both used Voigt functions with fixed Gaussian width for the detector resolution to fit the kaonic hydrogen lines with the following parameters: intensities of $K_{\alpha}, K_{\beta}$, and $K_{\gamma}$, energy of $K_{\alpha}$, one Lorentzian width for all $K$ lines, as the hadronic broadening comes from the ground-state width only. The energy differences between $K_{\alpha}$ and $K_{\beta}, K_{\gamma}, K_{\text {high }}$ were fixed to their electromagnetic values.

The two analysis methods differ essentially in the background spectrum which was used. Analysis I used the bulk of no-collision data as background spectrum. A simultaneous fit of the two spectra, kaonic hydrogen and nocollision, was performed by constructing a $\chi^{2}$ function as sum of the two individual ones. The same shape (a quadratic polynomial) of the continuous background compo- 
nent, apart from a normalization factor, was used to fit both spectra. All the fluorescence lines were normalized with the same factor. The fit range was selected to be from 2.9 to $10.4 \mathrm{keV}$, excluding the $K_{\text {high }}$ energy range (7.74$8.82 \mathrm{keV}$ ), in order to avoid model dependency as far as the intensities were concerned. The systematic errors introduced by these cuts were studied by Monte Carlo simulations and included in the global systematic error.

Analysis II used as a background spectrum the sum of kaonic nitrogen data [13] and a subset (low CCD occupancy) of no-collision data. By means of activation analyses performed in the laboratory it was checked that the silicon and iron fluorescence peaks do have the same origin (CCD itself and its support) in both signal and background spectra. Consequently, the intensity ratio of the silicon fluorescence peaks with respect to the integrated continuous background in the two spectra, described by two independent cubic polynomials, was used to normalize the iron fluorescence peak in the kaonic hydrogen spectrum.

Concerning the influence of the $K_{\text {high }}$ transitions, the fit range was restricted to a region where this contribution is marginal, given only by their low-energy tails. In order to study this contribution, different values for the $K_{\text {high }} / K_{\gamma}$ yields ratios, obtained from cascade calculations [15], were imposed in the fit. The effect of varying the yields ratios as well as the fit energy range was studied and included in the systematic error.

The two analyses gave consistent results. In both, the fit without kaonic hydrogen contribution gave a $\chi^{2} / \mathrm{NDOF}=$

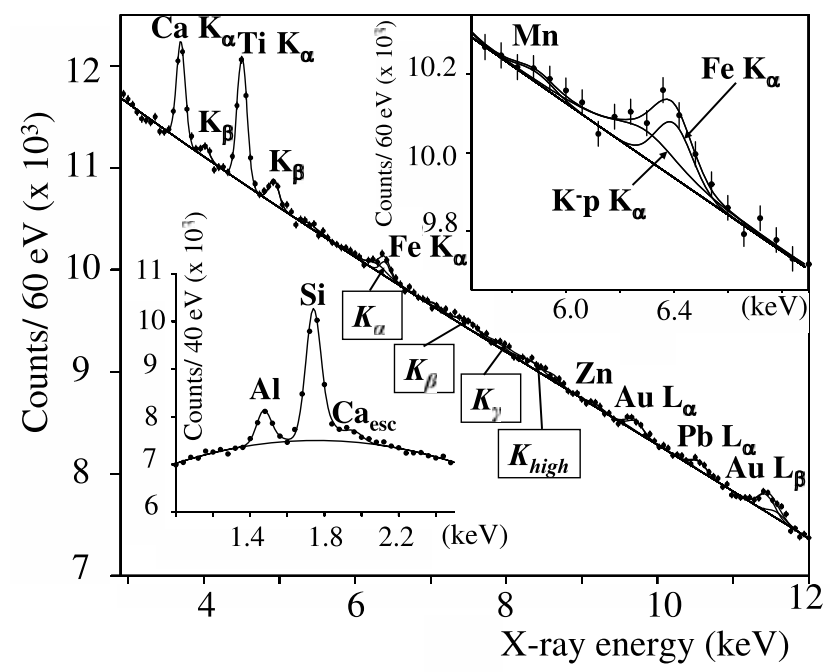

FIG. 1. The measured kaonic hydrogen x-ray spectrum. The kaonic hydrogen transitions (indicated by boxes) and the electronic excitations coming from setup materials are visible. The fit curves (total and components) are shown. Upper-right inset: zoom of the kaonic hydrogen $K_{\alpha}$ line region with the continuous background; the iron $K_{\alpha}$ and the kaonic hydrogen $K_{\alpha}$ lines disentangled. Lower-left inset: zoom of the silicon peak region with the aluminum, silicon, and calcium escape peaks.
1.25 where NDOF indicate the number of degrees of freedom. By including $\left(K^{-} p\right)$ lines, in analysis $\mathrm{I} \chi^{2} / \mathrm{NDOF}$ turned out to be 1.04 , and in analysis II the value was 1.02 . In Fig. 1, the fitted kaonic hydrogen x-ray spectrum is shown (spectra look the same in both analyses). A zoom of the $\left(K^{-} p\right) K_{\alpha}$ region shows the disentangling of the $\mathrm{Fe}$ $K_{\alpha}$ and $\left(K^{-} p\right) K_{\alpha}$ lines. In Fig. 2, the fitted measured background spectra used in the two analyses are shown. Figure 3 shows the kaonic hydrogen $\mathrm{x}$-ray spectrum after continuous and structured background subtraction for both analyses. The fitting curves of the various kaonic hydrogen lines are as well shown. Shifts and widths obtained in the two analyses are reported.

The weighted average of the two independent analyses for the $K_{\alpha}$ transition energy gave $6287 \pm 37$ (stat) \pm 6 (syst) $\mathrm{eV}$, where the statistical error is the smaller of the two individual ones.

The pure electromagnetic value of the kaonic hydrogen $K_{\alpha} \mathrm{x}$ ray is $6480 \pm 1 \mathrm{eV}$. Hence, the resulting ground-state shift $\epsilon_{1 s}$ is

$$
\epsilon_{1 s}=-193 \pm 37 \text { (stat) } \pm 6 \text { (syst) eV. }
$$

The weighted $1 s$ strong interaction width (FWHM) $\Gamma_{1 s}$ is

$$
\Gamma_{1 s}=249 \pm 111 \text { (stat) } \pm 30 \text { (syst) eV. }
$$

Systematic error contributions from detector energy calibration and energy resolution, as well as from analysis (fit range and method), were included.

Our result is consistent with the KEK measurement [11]

$$
\begin{aligned}
& \epsilon_{1 s}=-323 \pm 63 \text { (stat) } \pm 11 \text { (syst) eV, } \\
& \Gamma_{1 s}=407 \pm 208 \text { (stat) } \pm 100 \text { (syst) eV, }
\end{aligned}
$$

within $1 \sigma$ of their respective errors and confirms the repulsive character of the $K^{-} p$ interaction at threshold.

They differ significantly, however, in three important aspects: (1) the uncertainty of the DEAR result is about 2 times smaller than that of the KEK values; (2) the absolute values of $\epsilon_{1 s}$ and $\Gamma_{1 s}$ are $40 \%$ lower, which points towards a less repulsive strong interaction; (3) DEAR observed the

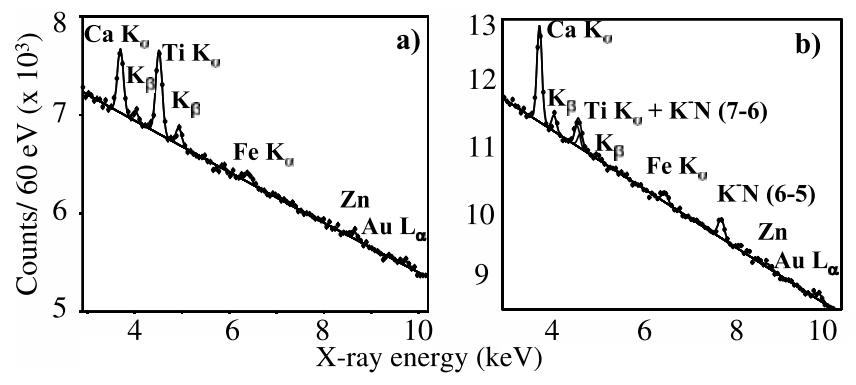

FIG. 2. The measured background spectra used in the two analysis methods: (a) no-collision data spectrum, in analysis I; (b) sum of the kaonic nitrogen data and of a subset (low CCD occupancy) of no-collision data, in analysis II. The fitting functions (overall peaks and continuous components) are shown. 

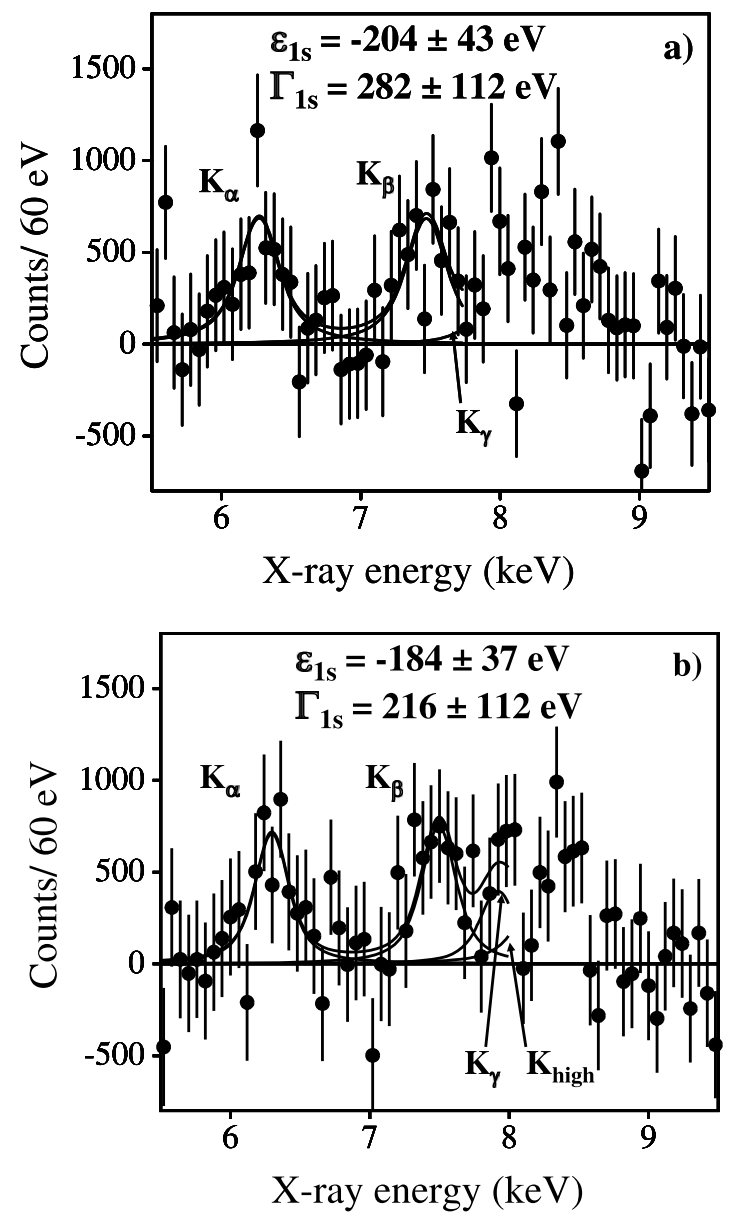

FIG. 3. The kaonic hydrogen x-ray spectrum after continuous and structured background subtraction: (a) results of analysis I; (b) results of analysis II. The fitting curves of the various kaonic hydrogen lines are shown.

pattern of kaonic hydrogen $K$ lines, clearly identifying $K_{\alpha}$, $K_{\beta}$, and $K_{\gamma}$ transitions, with an intensity ratio $1.1 \pm 0.5$ for $K_{\alpha} / K_{\beta}$ (compatible with [15] within $2 \sigma$ ), and limiting the intensity of $K_{\gamma} / K_{\alpha}$ to $0.2 \pm 0.5$.

A new precise value of the $K^{-} p$ scattering length $a_{K^{-}} p$ can be extracted from the DEAR result by using the Deser relation [10]:

$$
\epsilon_{1 s}+i \frac{\Gamma_{1 s}}{2}=2 \alpha^{3} \mu^{2} T_{K^{-} p}^{\mathrm{th}},
$$

where $\alpha$ is the fine structure constant, $\mu$ is the reduced mass of the $K^{-} p$ system, and $T_{K^{-}}^{\text {th }}$ is the $K^{-} p \rightarrow K^{-} p$ threshold amplitude, i.e., the zero-energy scattering length $a_{K^{-}}$. In the isospin limit, i.e., in the absence of the electromagnetic interaction and at $m_{d}=m_{u}, a_{K^{-}}{ }$can be expressed in terms of the scattering lengths for isospin $I=$ 0 and $I=1, a_{0}$ and $a_{1}$ :

$$
a_{K^{-} p}=\frac{1}{2}\left(a_{0}+a_{1}\right) .
$$

Recent work based on the nonrelativistic effective Langrangian approach to bound states [16] shows that in the case of kaonic hydrogen the isospin breaking corrections to the lowest order relation given by Eq. (6) are important, much larger than their counterparts in pionic hydrogen, or in pionium (typically, a few percent). Here, following the customary practice, we report $a_{K^{-}}$, at the lowest order, as given by Eqs. (5) and (6):

$$
\begin{aligned}
a_{K^{-} p}= & (-0.468 \pm 0.090(\text { stat }) \pm 0.015(\text { syst })) \\
& +i(0.302 \pm 0.135 \text { (stat) } \pm 0.036(\text { syst })) \mathrm{fm} .
\end{aligned}
$$

The DEAR collaboration wishes to thank the DAФNE machine crew for their friendly cooperation. This research was partially supported by the European CommunityAccess to Research Infrastructures, contract no. HPRICT 1999-00088, and partially supported by the Swiss National Science Foundation.

[1] Y. Nambu, Phys. Rev. Lett. 4, 380 (1960); J. Goldstone, Nuovo Cimento 19, 154 (1961); J. Goldstone, A. Salam, and S. Weinberg, Phys. Rev. 127, 965 (1962).

[2] R. Dashen, Phys. Rev. 183, 1245 (1969).

[3] For a review, see, for example, J.F. Donoghue, E. Golowich, and B. R. Holstein, Dynamics of the Standard Model (Cambridge University Press, Cambridge, 1992).

[4] E. Reya, Rev. Mod. Phys. 46, 545 (1974); H. Pagels, Phys. Rep. 16, 219 (1975).

[5] R. L. Jaffe and C. L. Korpa, Comments Nucl. Part. Phys. 17, 163 (1987).

[6] G. E. Brown and M. Rho, Phys. Rep. 363, 85 (2002).

[7] J. Gasser and M.E. Sainio, hep-ph/0002283; P. M. Gensini, hep-ph/9804344; A. D. Martin and G. Violini, Lett. Nuovo Cimento Soc. Ital. Fis. 30, 105 (1981); B. Di Claudio, G. Violini,and A. M. Rodriguez-Vargas, Lett. Nuovo Cimento Soc. Ital. Fis. 26, 555 (1979).

[8] K. F. Liu et al., Phys. Rev. D 59, 112001 (1999).

[9] C.-H. Lee, Phys. Rep. 275, 255 (1996); T. Inoue, V.E. Lyubovitskij, Th. Gutsche, and A. Faessler, Phys. Rev. C 69, 035207 (2004).

[10] S. Deser, M. L. Goldberger, K. Baumann, and W. Thirring, Phys. Rev. 96, 774 (1954).

[11] M. Iwasaki et al., Phys. Rev. Lett. 78, 3067 (1997); T. M. Ito et al., Phys. Rev. C 58, 2366 (1998).

[12] S. Bianco et al., Riv. Nuovo Cimento 22, 1 (1999).

[13] T. Ishiwatari et al., Phys. Lett. B 593, 48 (2004).

[14] V. Lucherini et al., Nucl. Instrum. Methods Phys. Res., Sect. A 496, 315 (2003).

[15] T. Jensen, DEAR Technical Note IR-46, 2003.

[16] U.-G. Meissner, U. Raha, and A. Rusetsky, Eur. Phys. J. C 35, 349 (2004). 